International Journal of Pattern Recognition

and Artificial Intelligence

Vol. 30, No. 10 (2016) 1699001 (14 pages)

(C) World Scientific Publishing Company

DOI: $10.1142 / S 0218001416990019$

\title{
Author Index \\ Volume 30 (2016)
}

Adeli, H., see Siddique, N.

30 (2016) 1639001

Alam, N., see Santosh, K. C.

30 (2016) 1657002

Alhussan, A. \& El Hindi, K., Selectively fine-tuning Bayesian network learning algorithm

30 (2016) 1651005

Aničić, O., see Jović, S.

Anjum, A., see Kausar, S.

30 (2016) 1659019

Antani, S., see Santosh, K. C.

30 (2016) 1650009

30 (2016) 1657002

Aravindan, C., see Mohanavalli, S.

30 (2016) 1650023

Bai, S., Hou, J. \& Ohnishi, N., Scene categorization through combining LBP and SIFT features effectively

30 (2016) 1655001

Benchaou, S., Nasri, M. \& El Melhaoui, O., New approach of features extraction for numeral recognition

Bian, K., see Zhou, X.

30 (2016) 1650014

Briand, C., see Mekonnen, A. A.

30 (2016) 1660007

30 (2016) 1655022

Cao, M., see Tan, G.

30 (2016) 1658001

Chao, M.-H., see Lin, S.-J.

30 (2016) 1655017

Chao, M.-T., see Chen, Y.-S.

30 (2016) 1654001

Chelly, Z. \& Elouedi, Z., From the general to the specific: Inducing a novel dendritic cell algorithm from a detailed state-of-the-art review

30 (2016) 1659009

Chen, D., see Tian, L.

30 (2016) 1655023

Chen, F., Hu, Z., Li, K. \& Liu, W., A hybrid skin detection model from multiple color spaces based on a dual-threshold Bayesian algorithm

30 (2016) 1655018

Chen, F., see $\mathrm{Yu}, \mathrm{B}$.

30 (2016) 1655013

Chen, J., see $\mathrm{Li}, \mathrm{X}$.

30 (2016) 1654006

Chen, L., see Guo, S.

30 (2016) 1650018

Chen, M., see Tong, Z.

30 (2016) 1660002

Chen, Q., see Liu, C.

30 (2016) 1658002

Chen, Q., see Xiao, D.

30 (2016) 1660005

Chen, W., see Qian, Y.

30 (2016) 1655010 
Chen, Y., Zhang, L. \& Yi, Z., A novel low rank representation algorithm for subspace clustering

Chen, Y.-S. \& Chao, M.-T., MAT-based thinning for line patterns

30 (2016) 1650007

Chen, Z., see $\mathrm{Li}, \mathrm{J}$.

30 (2016) 1654001

Chen, Z., see Wu, Q. E.

30 (2016) 1656005

30 (2016) 1656002

Chen, Z., Wang, K. \& Liu, C., Fast face sketch-photo image synthesis and recognition

30 (2016) 1656008

Cheng, H., see Peng, C.

30 (2016) 1651001

Cheng, W., see $\mathrm{Wu}, \mathrm{Q}$. E.

30 (2016) 1656002

Cheng, Y. \& Yu, H., Adaptive group consensus of multi-agent networks via pinning control

30 (2016) 1659014

Choi, J. Y., Feature subspace estimation for resolving dimensionality mismatch in practical color-based face recognition systems

Coenen, F., see Xia, Y.

30 (2016) 1656004

30 (2016) 1660010

Coenen, F., see Yan, C.

30 (2016) 1650010

Cortés, X. \& Serratosa, F., Learning graph matching substitution weights based on the ground truth node correspondence

Cui, Z., see Li, X.

Dai, Y., Wang, H. \& Wang, Y., Chaotic medical image encryption algorithm based on bit-plane decomposition

Danesh, A. S., see Jović, S.

Dawson-Howe, K., see Fitzsimons, J.

Deng, A., see Zhang, G.-D.

Deng, M., Tian, F., Ran, J., Shi, Z. \& Zhang, L., A novel frame rate up-conversion algorithm based on soft threshold bandelet transform

(2016) 1650017

Deris, M. M., see Lasisi, A.

Dimopoulos, A. C., see Pavlatos, C.

Ding, F., see Zhao, Y.

30 (2016) 1650005

30 (2016) 1654006

30 (2016) 1657001

30 (2016) 1659019

30 (2016) 1655002

30 (2016) 1660011

Du, S., see Liu, Q.

Du, Y., see Wu, Q. E.

Duan, X., see Huang, T.

El Hindi, K., see Alhussan, A.

El Melhaoui, O., see Benchaou, S.

Elouedi, Z., see Chelly, Z.

Erbilek, M., see Liang, Y.

Fairhurst, M. C., see Liang, Y.

Fan, L., Meng, S., Liu, X. \& Liang, Y., Improved CTT-SP algorithm with critical path method for massive data storage in scientific workflow systems

30 (2016) 1659023

Fan, L., see Ouyang, A.

30 (2016) 1659012 
Fayek, M., see Negied, N. K. A.-W.

30 (2016) 1655025

Feng, L., see Yin, C.

30 (2016) 1659013

Fink, G. A., see Grzeszick, R.

30 (2016) 1655005

Fitzsimons, J. \& Dawson-Howe, K., Abandoned, removed and moved object classification

$\mathrm{Fu}, \mathrm{D}$., see Yang, $\mathrm{T}$.

Fujimura, T., see Sugimura, D.

Fuller, E., see Qi, X.

Gao, C., see Guo, S.

Gao, Y., see Wang, X.

Ghassemian, H., see Hosseini, S. A.

Ghazali, R., see Lasisi, A.

Grossi, G., Lanzarotti, R. \& Lin, J., Robust face recognition providing the identity and its reliability degree combining sparse representation and multiple features

Grzeszick, R. \& Fink, G. A., An iterative partitioning-based method for semi-supervised annotation learning in image collections

30 (2016) 1655002

30 (2016) 1650022

30 (2016) 1655009

30 (2016) 1650006

30 (2016) 1650018

30 (2016) 1654005

30 (2016) 1650001

30 (2016) 1660008

30 (2016) 1656007

Gu, F., see Liu, H.-L.

$\mathrm{Gu}, \mathrm{F}$., see Luo, J.

Guest, R. M., see Liang, Y.

Guo, S., Pan, H., Tan, G., Chen, L. \& Gao, C., A high invariance motion representation for skeleton-based action recognition

Guo, S.-N., see Ma, J.-M.

Guo, Y., see Wan, J.

Guo, Z., Zhou, Q., Liu, Z. \& Liu, C., Accurate pupil center location with the SIFT descriptor and SVM classifier

Hamamoto, T., see Sugimura, D.

Han, J., see Wang, W.

Han, L., see Zhou, X.

Han, R., see Wu, Q. E.

Han, Y., see Wang, X.

Hashemi, S., see Tahmoresnezhad, J.

He, G., see Wang, X.

Hei, X., see Zhang, C.

Hemayed, E. B., see Negied, N. K. A.-W.

Herawan, T., see Lasisi, A.

Herbulot, A., see Mekonnen, A. A.

Hong, H., see Zhou, X.

30 (2016) 1655005

30 (2016) 1659002

30 (2016) 1659007

30 (2016) 1653001

30 (2016) 1650018

30 (2016) 1659025

30 (2016) 1650011

30 (2016) 1655012

30 (2016) 1655009

30 (2016) 1659008

30 (2016) 1660007

30 (2016) 1656002

30 (2016) 1655008

30 (2016) 1651003

30 (2016) 1655008

30 (2016) 1659003

30 (2016) 1655025

30 (2016) 1660008

30 (2016) 1655022

30 (2016) 1660007

Hosseini, S. A. \& Ghassemian, H., Hyperspectral data feature extraction using rational function curve fitting

30 (2016) 1650001

Hou, J., see Bai, S.

30 (2016) 1655001 
Hou, L., see Wen, Y.

30 (2016) 1656003

Hsu, H.-C., see Lo, R.-C.

30 (2016) 1655021

$\mathrm{Hu}, \mathrm{Q}$, see Wan, J.

30 (2016) 1650011

$\mathrm{Hu}$, Y., see Qin, L.

30 (2016) 1655019

$\mathrm{Hu}, \mathrm{Z}$., see Chen, F.

30 (2016) 1655018

Huang, G., see Liu, Z.

30 (2016) 1656001

Huang, T., Zhang, S., Duan, X. \& Liang, R., On-line handy handwriting chinese characters input for non-chinese speakers based on wavelet neural network

30 (2016) 1659017

Huang, W., Wang, X., Zhu, Y. \& Zheng, G., Improved LRC based on combined virtual training samples for face recognition

Hui, J., see Liu, Q.

Huo, Y., see Shen, N.-M.

Jaisakthi, S. M., see Mohanavalli, S.

Jalalirad, A. \& Tjalkens, T., An efficient method for computing a Bayesian mixture of feature-based models

Javed, M. Y., see Kausar, S.

Jiang, B., see Wei, Q.

Jiang, L., see Zhang, L.

Jiang, X. L., Li, B. L., Yuan, J. Y. \& Wu, X. L., Active contour driven by local Gaussian distribution fitting and local signed difference based on local entropy

30 (2016) 1655011

Jiao, L., see Shang, R.

30 (2016) 1659024

Jie, G., see Tan, G.

30 (2016) 1658001

Jing, L., see Shi, H.

30 (2016) 1650002

Jović, S., Danesh, A. S., Younesi, E., Aničić, O., Petković, D. \& Shamshirband, S., Forecasting of underactuated robotic finger contact forces by support vector regression methodology

Kausar, S., Javed, M. Y., Tehsin, S. \& Anjum, A., A novel mathematical modeling and parameterization for sign language classification

Kim, D., see Lee, G.

1650009

Ko, M., see Peng, C.

(2016) 1650012

Lane, I., see Yu, B.

30 (2016) 1651001

30 (2016) 1655013

Lanzarotti, R., see Grossi, G.

30 (2016) 1656007

Lasisi, A., Ghazali, R., Deris, M. M., Herawan, T. \& Lasisi, F., Extracting information in agricultural data using fuzzy-rough sets hybridization and clonal selection theory inspired algorithms

30 (2016) 1660008

Lasisi, F., see Lasisi, A.

30 (2016) 1660008

Lee, C.-Y., see Lin, S.-J.

30 (2016) 1655017 
Lee, G., Yun, U., Ryang, H. \& Kim, D., Approximate maximal frequent pattern mining with weight conditions and error tolerance

Lerasle, F., see Mekonnen, A. A.

Li, B. L., see Jiang, X. L.

Li, C., see Zhang, L.

Li, G., Wang, L. \& Ou, W., Robust personalized ranking from implicit feedback

Li, H., Ma, X.-P. \& Shi, J., Incorporating trust relation with PMF to enhance social network recommendation performance

Li, H., see Liu, C.

Li, H. \& Wang, Z., An evolutionary algorithm using parameter space searching for interval linear fractional bilevel programming problems

Li, J., Chen, Z. \& Liu, C., Low-resolution face recognition of multi-scale blocking CS-LBP and weighted PCA

Li, J., see Shen, N.-M.

Li, K., see Ouyang, A.

Li, K., see Chen, F.

Li, K., see Xiao, G.

Li, S., see Xiao, D.

Li, X., Chen, J., Cui, Z., Wu, M. \& Zhu, X., Single image superresolution based on sparse representation with adaptive dictionary selection

Li, Y. S., see Tu, C. T.

Liang, H., see Liu, C.

Liang, R., see Huang, T.

Liang, Y., Fairhurst, M. C., Guest, R. M. \& Erbilek, M., Automatic handwriting feature extraction, analysis and visualization in the context of digital palaeography

Liang, Y., see Fan, L.

Lin, H. W., see Tu, C. T.

Lin, H.-J., see Tu, C. T.

Lin, J., see Grossi, G.

Lin, S.-J., Chao, M.-H., Lee, C.-Y. \& Yang, C.-S., Human action recognition using motion history image based temporal segmentation

Lipikorn, R., see Srichumroenrattana, N.

Liu, C., Chen, Q., Liang, H. \& Li, H., Digital watermarking processing technique based on overcomplete dictionary

Liu, C., see Chen, Z.

Liu, C., see Guo, Z.
30 (2016) 1650012

30 (2016) 1655022

30 (2016) 1655011

30 (2016) 1650003

30 (2016) 1659001

30 (2016) 1659016

30 (2016) 1658002

30 (2016) 1659011

30 (2016) 1656005

30 (2016) 1655003

30 (2016) 1659012

30 (2016) 1655018

30 (2016) 1660001

30 (2016) 1660005

30 (2016) 1654006

30 (2016) 1655015

30 (2016) 1658002

30 (2016) 1659017

30 (2016) 1653001

30 (2016) 1659023

30 (2016) 1655015

30 (2016) 1655015

30 (2016) 1656007

30 (2016) 1655017

30 (2016) 1655006

30 (2016) 1658002

30 (2016) 1656008

30 (2016) 1655012 
Liu, C., see Li, J.

30 (2016) 1656005

Liu, C., see Yue, G.-D.

30 (2016) 1650020

Liu, H., see Tong, Z.

30 (2016) 1660002

Liu, H.-L., Peng, C., Gu, F. \& Wen, J., A constrained multiobjective evolutionary algorithm based on boundary search and archive

30 (2016) 1659002

Liu, H.-Y., see Wang, Q.

30 (2016) 1655007

Liu, J., see Wan, J.

30 (2016) 1650011

Liu, Q., Hui, J., Luo, L. \& Yang, Y., Target identification and location algorithm based on SURF-BRISK operator

30 (2016) 1655016

Liu, Q., van Wyk, B. J., Du, S. \& Sun, Y., Dynamic small world network topology for particle swarm optimization

30 (2016) 1660009

Liu, R. \& Tao, J., An inference methodology for selecting and clustering genes based on likelihood ratio test

30 (2016) 1650019

Liu, S., see Zhang, Z.

30 (2016) 1655014

Liu, T., see Wang, $\mathrm{P}$.

30 (2016) 1659020

Liu, W., see Chen, F.

30 (2016) 1655018

Liu, X., see Fan, L.

Liu, Y., Error awareness by lower and upper bounds in ensemble learning

30 (2016) 1659023

30 (2016) 1660003

Liu, Y., see Ouyang, A.

Liu, Z., Pu, J., Qiu, Y., Zhang, M., Zhang, X. \& Huang, G., A

30 (2016) 1659012 kernel two-phase test sample sparse representation for face recognition

30 (2016) 1656001

Liu, Z., see Guo, Z.

30 (2016) 1655012

Lo, R.-C. \& Hsu, H.-C., A circular band extraction method based on extended Hough transform

30 (2016) 1655021

Luo, C.-H., see Wang, Q.

30 (2016) 1655007

Luo, J. \& Gu, F., An adaptive niching-based evolutionary algorithm for optimizing multi-modal function

30 (2016) 1659007

Luo, L., see Liu, Q.

30 (2016) 1655016

Luo, R., see Qi, X.

30 (2016) 1650006

Luo, Z., see Sun, Q.

30 (2016) 1659015

Lursinsap, C., see Srichumroenrattana, N.

30 (2016) 1655006

Ma, J.-M., Guo, S.-N., Su, R.-J. \& Yue, W.-N., The method for magnetic hyperthermia based on particle swarm optimization algorithm with levy flight

30 (2016) 1659025

Ma, L., see Yin, C.

30 (2016) 1659013

Ma, Q., see Wang, Q.

30 (2016) 1655007

Ma, X.-P., see Li, H.

30 (2016) 1659016 
Mekonnen, A. A., Lerasle, F., Herbulot, A. \& Briand, C., Incorporating computation time measures during heterogeneous features selection in a boosted cascade people detector

30 (2016) 1655022

Meng, S., see Fan, L.

Mohanavalli, S., Jaisakthi, S. M. \& Aravindan, C., Automatic scale parameters in affinity matrix construction for improved spectral clustering

Muneeswaran, K., see Rani, P. I.

Nagaraju, C., see Pemula, R.

Nasri, M., see Benchaou, S.

Negied, N. K. A.-W., Hemayed, E. B. \& Fayek, M., HSBS: A human's heat signature and background subtraction hybrid approach for crowd counting and analysis

Ni, D., see Tian, L.

Ohnishi, N., see Bai, S.

$\mathrm{Ou}, \mathrm{W}$., see Li, G.

Ouyang, A., Peng, X., Liu, Y., Fan, L. \& Li, K., An efficient hybrid algorithm based on HS and SFLA

Özöğür Akyüz, S., Üstünkar, G. \& Weber, G. W., Adapted infinite kernel learning by multi-local algorithm

Pan, H., see Guo, S.

Papakonstantinou, G., see Pavlatos, C.

Pavlatos, C., Dimopoulos, A. C. \& Papakonstantinou, G., Parallel hardware stochastic context-free parsers

Pemula, R. \& Nagaraju, C., Generation of random fields for image segmentation using manifold learning technique

Peng, C., Cheng, H. \& Ko, M., An efficient two-stage sparse representation method

Peng, C., see Liu, H.-L.

Peng, X., see Ouyang, A.

Peng, Z., Wang, Y., Xue, X. \& Wei, J., An efficient algorithm for suffix sorting

Petković, D., see Jović, S.

$\mathrm{Pu}$, J., see Liu, Z.

Qi, X., Luo, R., Fuller, E., Luo, R. \& Zhang, C.-Q., Signed quasi-clique merger: A new clustering method for signed networks with positive and negative edges

Qian, Y., Chen, W. \& Shen, I., Action recognition from pose signature in static image

Qiao, Y., see Sun, Q.

Qin, J., see Wang, X.
30 (2016) 1659023

30 (2016) 1650023

30 (2016) 1655020

30 (2016) 1654007

30 (2016) 1650014

30 (2016) 1655025

30 (2016) 1655023

30 (2016) 1655001

30 (2016) 1659001

30 (2016) 1659012

30 (2016) 1651004

30 (2016) 1650018

30 (2016) 1650008

30 (2016) 1650008

30 (2016) 1654007

30 (2016) 1651001

30 (2016) 1659002

30 (2016) 1659012

30 (2016) 1659018

30 (2016) 1659019

30 (2016) 1656001

30 (2016) 1650006

30 (2016) 1655010

30 (2016) 1655026

30 (2016) 1654005 
Qin, L., Wang, T., Hu, Y. \& Yao, C., Improved position and attitude determination method for monocular vision in vehicle collision warning system

Qiu, Y., see Liu, Z.

Ragab, K., Fast and parallel summed area table for fabric defect detection

Rama, R., see Sureshkumar, W.

Ran, J., see Deng, M.

Rani, P. I. \& Muneeswaran, K., Facial emotion recognition based on eye and mouth regions

Ren, G., see Wan, J.

Ren, T., see Yue, G.-D.

Roy, P. P., see Santosh, K. C.

Ryang, H., see Lee, G.

Santosh, K. C., Alam, N., Roy, P. P., Wendling, L., Antani, S. \& Thoma, G. R., A simple and efficient arrowhead detection technique in biomedical images

Serratosa, F., see Cortés, X.

Shamshirband, S., see Jović, S.

Shang, R., Zhang, W. \& Jiao, L., Circularly searching core nodes based label propagation algorithm for community detection

Sharet, N. \& Shimshoni, I., Analyzing data changes using mean shift clustering

Shen, I., see Qian, Y.

Shen, N.-M., Li, J., Zhou, P.-Y., Huo, Y. \& Zhuang, Y., BSFCoS: Block and sparse principal component analysisbased fast co-saliency detection method

Sheng, V. S., see Yan, X.

Shi, H., Zhao, X., Zhen, L. \& Jing, L., Twin bounded support tensor machine for classification

Shi, J., see Li, H.

Shi, X., see Wang, L.

Shi, Z., see Deng, M.

Shih, F. Y. \& Zhong, X., Intelligent watermarking for highcapacity low-distortion data embedding

Shimshoni, I., see Sharet, N.

Siddique, N. \& Adeli, H., Gravitational search algorithm and its variants

Song, A., see Tian, L.

Srichumroenrattana, N., Lipikorn, R. \& Lursinsap, C., Stereoscopic face reconstruction from a single 2-dimensional face image using orthogonality of normal surface and Y-ratio
30 (2016) 1655019

30 (2016) 1656001

30 (2016) 1660004

30 (2016) 1650004

30 (2016) 1650017

30 (2016) 1655020

30 (2016) 1650011

30 (2016) 1650020

30 (2016) 1657002

30 (2016) 1650012

30 (2016) 1657002

30 (2016) 1650005

30 (2016) 1659019

30 (2016) 1659024

30 (2016) 1650016

30 (2016) 1655010

30 (2016) 1655003

30 (2016) 1650013

30 (2016) 1650002

30 (2016) 1659016

30 (2016) 1659021

30 (2016) 1650017

30 (2016) 1654003

30 (2016) 1650016

30 (2016) 1639001

30 (2016) 1655023

30 (2016) 1655006 
Su, R.-J., see Ma, J.-M.

30 (2016) 1659025

Sugimura, D., Fujimura, T. \& Hamamoto, T., Enhanced cascading classifier using multi-scale HOG for pedestrian detection from aerial images

30 (2016) 1655009

Sun, Q., Qiao, Y., Wu, H. \& Wang, J., An edge detection method based on adjacent dispersion

30 (2016) 1655026

Sun, Q., Wang, N., Zhou, Y. \& Luo, Z., Identification of influential online social network users based on multi-features

30 (2016) 1659015

Sun, Y., see Liu, Q.

Sureshkumar, W. \& Rama, R., Chomsky hierarchy control on isotonic array $\mathrm{P}$ systems

30 (2016) 1660009

30 (2016) 1650004

Tahmoresnezhad, J. \& Hashemi, S., An efficient yet effective random partitioning and feature weighting approach for transfer learning

Tan, G., Jie, G. \& Cao, M., A new method of sound quality analysis based on design of experiment

Tan, G., see Guo, S.

Tan, X., see Wei, Q.

Tang, C., see Wang, X.

Tao, J., see Liu, R.

Tehsin, S., see Kausar, S.

Thoma, G. R., see Santosh, K. C.

Tian, F., see Deng, M.

Tian, L., Song, A., Chen, D. \& Ni, D., Haptic display of image based on multi-feature extraction

30 (2016) 1655023

Tian, M.-L. \& Yang, J.-M., Optimization of structural element in froth image denoising of coal flotation

30 (2016) 1654002

Tjalkens, T., see Jalalirad, A.

30 (2016) 1651002

Tong, Z., Xiao, Z., Liu, H. \& Chen, M., A novel parallel LSA-SVM algorithm based on semantic distance for blog

Tu, C. T., Lin, H. W., Lin, H.-J. \& Li, Y. S., Super-resolution based on clustered examples

30 (2016) 1651003

30 (2016) 1658001

30 (2016) 1650018

30 (2016) 1659022

30 (2016) 1655008

30 (2016) 1650019

30 (2016) 1650009

30 (2016) 1657002

30 (2016) 1650017

Urio, P. R., Verri, F. A. N. \& Zhao, L., Semi-supervised classification by particle competition in complex network's edges

Üstünkar, G., see Özöğür Akyüz, S.

1660006

30 (2016) 1651004

30 (2016) 1660009

van Wyk, B. J., see Liu, Q.

30 (2016) 1660006

Wan, J., Liu, J., Ren, G., Guo, Y., Yu, D. \& Hu, Q., Day-ahead prediction of wind speed with deep feature learning

30 (2016) 1650011

Wang, C., see Zhang, Z.

30 (2016) 1655014

Wang, H., see Dai, Y.

30 (2016) 1657001 
Wang, H., see Zhu, H.

30 (2016) 1659006

Wang, J., see Sun, Q.

30 (2016) 1655026

Wang, K., see Chen, Z.

30 (2016) 1656008

Wang, K., see Zhou, L.

30 (2016) 1650015

Wang, L., see Li, G.

30 (2016) 1659001

Wang, L., see Wei, Q.

30 (2016) 1659022

Wang, L., see Yue, G.-D.

30 (2016) 1650020

Wang, L., see Zhang, C.

30 (2016) 1659003

Wang, L., Zhang, L., Xu, M. \& Shi, X., Research on fault diagnosis method of civil aviation engine variable bleed valve system based on artificial immune algorithm

30 (2016) 1659021

Wang, M., Wang, Y. \& Wang, X., A space division multiobjective evolutionary algorithm based on adaptive multiple fitness functions

Wang, N., see Sun, Q.

Wang, P., Zhang, C., Zhang, B., Liu, T. \& Wu, J., A dimensional diversity based hybrid multiobjective evolutionary algorithm for optimization problem

30 (2016) 1659020

Wang, Q., Ma, Q., Luo, C.-H., Liu, H.-Y. \& Zhang, C.-L., Hybrid histogram of oriented optical flow for abnormal behavior detection in crowd scenes

30 (2016) 1655007

Wang, R., see Zhou, Y.

Wang, S., see Wang, X.

Wang, T., see Qin, L.

Wang, W., Han, J., Zheng, T., Zheng, G. \& Zhou, X., Speaker verification via modeling Kurtosis using sparse coding

Wang, X., He, G., Tang, C., Han, Y. \& Wang, S., Keypointsbased image passive forensics method for copy-move attacks

Wang, X., Qin, J. \& Gao, Y., Artistic coloring: Color transfer from painting

Wang, X., see Huang, W.

30 (2016) 1659010

30 (2016) 1655008

30 (2016) 1655019

30 (2016) 1659008

30 (2016) 1655008

30 (2016) 1654005

Wang, X., see Wang, M.

30 (2016) 1656006

30 (2016) 1659005

Wang, Y., see Dai, Y.

30 (2016) 1657001

Wang, Y., see Peng, Z.

30 (2016) 1659018

Wang, Y., see Wang, M.

30 (2016) 1659005

Wang, Z., see Li, H.

30 (2016) 1659011

Wang, Z., see Zhou, G.

Weber, G. W., see Özöğür Akyüz, S.

30 (2016) 1654004

30 (2016) 1651004

Wei, J., see Peng, Z.

30 (2016) 1659018

Wei, Q., Tan, X., Jiang, B. \& Wang, L., Evacuating from an unknown affected area

30 (2016) 1659022

Wei, Y., see Zhou, G.

30 (2016) 1654004 
Wen, J., see Liu, H.-L.

30 (2016) 1659002

Wen, Y., Zhang, L. \& Hou, L., Discriminant sparsity preserving analysis for face recognition

30 (2016) 1656003

Wendling, L., see Santosh, K. C.

30 (2016) 1657002

$\mathrm{Wu}, \mathrm{C}$., see Yang, $\mathrm{T}$.

$\mathrm{Wu}, \mathrm{H}$., see Sun, Q.

30 (2016) 1650022

30 (2016) 1655026

$\mathrm{Wu}$, J., see Wang, $\mathrm{P}$.

30 (2016) 1659020

$\mathrm{Wu}, \mathrm{M}$., see $\mathrm{Li}, \mathrm{X}$.

30 (2016) 1654006

Wu, Q., see Yan, X.

30 (2016) 1650013

Wu, Q. E., Chen, Z., Han, R., Yang, C., Du, Y., Zheng, Y. \& Cheng, W., A palmprint recognition approach based on image segmentation of region of interest

Wu, X. L., see Jiang, X. L.

Xia, Y., Zhang, B. \& Coenen, F., Face occlusion detection using deep convolutional neural networks

Xiang, X., see Zhao, Y.

Xiao, B., see Zhang, Z.

Xiao, D., Chen, Q. \& Li, S., A multi-scale cascaded hierarchical model for image labeling

Xiao, G., Li, K., Zhou, X. \& Li, K., Queueing analysis of continuous queries for uncertain data streams over sliding windows

Xiao, Z., see Tong, Z.

Xie, K., see Zhou, X.

Xing, X., see Zhou, X.

$\mathrm{Xu}, \mathrm{B}$., see Zhang, $\mathrm{T}$.

$\mathrm{Xu}, \mathrm{M}$. , see Wang, L.

$\mathrm{Xu}, \mathrm{Z}$., see Yue, G.-D.

Xur, X., see Peng, Z.

Yan, C., Coenen, F., Yue, Y., Yang, X. \& Zhang, B., Videobased classification of driving behavior using a hierarchical classification system with multiple features

30 (2016) 1656002

30 (2016) 1655011

30 (2016) 1660010

30 (2016) 1655024

30 (2016) 1655014

30 (2016) 1660005

30 (2016) 1660001

30 (2016) 1660002

30 (2016) 1660007

30 (2016) 1660007

30 (2016) 1655004

30 (2016) 1659021

30 (2016) 1650020

30 (2016) 1659018

Yan, R., Combining Renyi entropy and EWMA to detect common attacks in network

30 (2016) 1650010

30 (2016) 1650021

Yan, X., Wu, Q. \& Sheng, V. S., A double weighted Naive Bayes with niching cultural algorithm for multi-label classification

30 (2016) 1650013

Yang, C., see Wu, Q. E.

30 (2016) 1656002

Yang, C.-S., see Lin, S.-J.

30 (2016) 1655017

Yang, D., see Zhang, C.

30 (2016) 1659003

Yang, G., see Zhou, L.

30 (2016) 1650015 
Yang, T., Fu, D. \& Wu, C., Laplacian embedded infinite kernel

30 (2016) 1650022 model for semi-supervised classification

Yang, X., see Yan, C.

30 (2016) 1650010

Yang, Y., see Liu, Q.

30 (2016) 1655016

Yao, C., see Qin, L.

30 (2016) 1655019

Yi, Z., see Chen, Y.

30 (2016) 1650007

Yin, C., Ma, L. \& Feng, L., A feature selection method for improved clonal algorithm towards intrusion detection

30 (2016) 1659013

Yin, Y., see Zhou, L.

30 (2016) 1650015

Younesi, E., see Jović, S.

30 (2016) 1659019

Yu, B., Lane, I. \& Chen, F., 3D face detection via reconstruction over hierarchical features for single face situations

30 (2016) 1655013

Yu, D., see Wan, J.

30 (2016) 1650011

Yu, H., see Cheng, Y.

Yu, L. \& Zhou, K., A dynamic local path planning method for

30 (2016) 1659014 outdoor robot based on characteristics extraction of laser rangefinder and extended support vector machine

30 (2016) 1659004 $\mathrm{Yu}, \mathrm{X}$., see Zhao, Y.

Yuan, J. Y., see Jiang, X. L.

Yue, G.-D., Xu, Z., Wang, L., Liu, C. \& Ren, T., WSN-Based

30 (2016) 1655024

30 (2016) 1655011 vibration characteristic research for various railway track structures for pattern classification

30 (2016) 1650020

Yue, W.-N., see Ma, J.-M.

30 (2016) 1659025

Yue, Y., see Yan, C.

30 (2016) 1650010

Yun, U., see Lee, G.

30 (2016) 1650012

Zeng, Y., see Zhang, T.

30 (2016) 1655004

Zhang, B., see Wang, $\mathrm{P}$.

30 (2016) 1659020

Zhang, B., see Xia, Y.

30 (2016) 1660010

Zhang, B., see Yan, C.

30 (2016) 1650010

Zhang, C., Hei, X., Yang, D. \& Wang, L., A memetic particle swarm optimization algorithm for community detection in complex networks

30 (2016) 1659003

Zhang, C., see Wang, P.

30 (2016) 1659020

Zhang, C.-L., see Wang, Q.

30 (2016) 1655007

Zhang, C.-Q., see Qi, X.

30 (2016) 1650006

Zhang, L., Jiang, L. \& Li, C., A new feature selection approach to Naive Bayes text classifiers

30 (2016) 1650003

Zhang, L., see Chen, Y.

30 (2016) 1650007

Zhang, L., see Deng, M.

30 (2016) 1650017

Zhang, L., see Wang, L.

30 (2016) 1659021 
Zhang, L., see Wen, Y.

30 (2016) 1656003

Zhang, M., see Liu, Z.

30 (2016) 1656001

Zhang, R., see Zhao, Y.

30 (2016) 1655024

Zhang, S., see Huang, $\mathrm{T}$.

30 (2016) 1659017

Zhang, T., Zeng, Y. \& Xu, B., HCNN: A neural network model for combining local and global features towards human-like classification

30 (2016) 1655004

Zhang, W., see Shang, R.

30 (2016) 1659024

Zhang, X., see Liu, Z.

30 (2016) 1656001

Zhang, Y. \& Deng, A., Redundancy reduction algorithms in rule-based knowledge bases

30 (2016) 1660011

Zhang, Z., Liu, S., Wang, C., Xiao, B. \& Zhou, W., Multiple continuous virtual paths based cross-view action recognition

Zhao, L., see Urio, P. R.

Zhao, X., see Shi, H.

Zhao, Y., Ding, F., Yu, X., Zhang, R. \& Xiang, X., A new waters hole detection and tracking method for UGV in cross-country environment

Zhen, L., see Shi, H.

Zheng, G., see Huang, W.

Zheng, G., see Wang, W.

Zheng, T., see Wang, W.

Zheng, Y., see Wu, Q. E.

Zhong, X., see Shih, F. Y.

Zhou, G., Zhu, D., Wei, Y. \& Wang, Z., Hybrid transmittance fitting for rendering transparency on the GPU

Zhou, K., see Yu, L.

Zhou, L., Yang, G., Yin, Y., Yang, L. \& Wang, K., Finger vein recognition based on stable and discriminative superpixels

Zhou, P.-Y., see Shen, N.-M.

Zhou, Q., see Guo, Z.

Zhou, W., see Zhang, Z.

Zhou, X., see Wang, W.

Zhou, X., see Xiao, G.

Zhou, X., Xing, X., Han, L., Hong, H., Bian, K. \& Xie, K.,

Structure feature learning method for incomplete data

Zhou, Y., see Sun, Q.

Zhou, Y. \& Wang, R., An improved flower pollination algorithm for optimal unmanned undersea vehicle path planning problem Zhu, D., see Zhou, G.

30 (2016) 1655014

30 (2016) 1660006

30 (2016) 1650002

30 (2016) 1655024

30 (2016) 1650002

30 (2016) 1656006

30 (2016) 1659008

30 (2016) 1659008

30 (2016) 1656002

30 (2016) 1654003

30 (2016) 1654004

30 (2016) 1659004

30 (2016) 1650015

30 (2016) 1655003

30 (2016) 1655012

30 (2016) 1655014

30 (2016) 1659008

30 (2016) 1660001

30 (2016) 1660007

30 (2016) 1659015

30 (2016) 1659010

30 (2016) 1654004 
Zhu, H. \& Wang, H., New deadline-aware energy-consumption optimization model and genetic algorithm under cloud computing

30 (2016) 1659006

Zhu, X., see Li, X.

30 (2016) 1654006

Zhu, Y., see Huang, W.

30 (2016) 1656006

Zhuang, Y., see Shen, N.-M.

30 (2016) 1655003 